\title{
Anomaly Stop Detection by Smartphone
}

\author{
Viet Chau Dang*, Masao Kubo, Hiroshi Sato, Tomohiro Shirakawa, Akira Namatame \\ Computer Science, National Defense Academy, Hashirimizu 1 \\ Yokosuka, Kanagawa, 239-8686, Japan \\ *Corresponding author. Email: dangvietchau@gmail.com \\ Tel:+81-46-841-3810,Fax:+81-46-844-5911
}

\begin{abstract}
This paper proposes a method for detecting anomaly stop events from GPS logs of a vehicle recorded by smartphone. Despite of many researches of strong braking event detection are proposed, they cannot discriminate between a normal and an abnormal braking event. Our proposal includes an IMAC model for GPS based map generation and an algorithm for anomaly stops detection based on the acquired map. The real world experiment shows our map generation method and its anomaly stop detection rule works well.
\end{abstract}

Keywords: Smartphone, Geographic Information System, Safety Map, Social Network Service, Anomaly Detection

\section{Introduction}

This paper studies a method for detecting anomaly stop events from GPS log of a vehicle which is recorded by a smartphone. Recently, there have been numerous researches of analysis transport data to extract and share useful information for improving safety of transport environment ${ }^{[1,2,3]}$. For example, Honda Company collects hard braking event data of a vehicle equipped with sensors, and shares the map over Internet for public use $^{[1]}$. We expect to collect data from community then we use smartphone as a terminal log data. For automatically generating safety map from driving log data, incidents which are important to drive safely have to be extracted from the log data. We choose stop motion events of vehicle as source of detection of incident. When driver encounters obstacles during driving for example, jump out of street, it usually stops the vehicle for accident avoidance.

Currently, there have been numerous studies on road environment estimation based on strong braking detection using probe car. However, they cannot discriminate between a normal and an abnormal braking event. In this paper, to detect anomaly stop events: 1) the system learns normal driving states from history; 2) anomalous stop events are detected by comparing the normal driving states and current driving states. In comparing to stop events caused by traffic signs and permanent road condition, anomalous stop caused by jump out of street doesn't frequently occur. The success of detecting anomalous stop at which there is no traffic sign is expected to contribute to the improvement of safety of traffic road system.

This paper proposes an extended version of IMAC $^{[5]}$ model for GPS based grid map generation. The IMAC model supposes that each grid is a two-state Markov 
graph which can be used to represent traffic elements such as traffic signal or semi-static objects. We use IMAC as a map generation method of a traffic environment.

The paper is organized as follows. Section 2 introduces our approach for GPS based grid map generation and an algorithm for anomalous stop detection. Section 3 shows the real world experiment with discussion on the results.

\section{Approach for Map Generation and Anomaly Detection}

This section denotes the method of occupancy grid map generation and its application for detecting anomalous stop. We first explain about the IMAC model ${ }^{[5]}$. The proposed algorithm is given in the next sub-sections: the grid update algorithm and the propose method for anomalous stop detection.

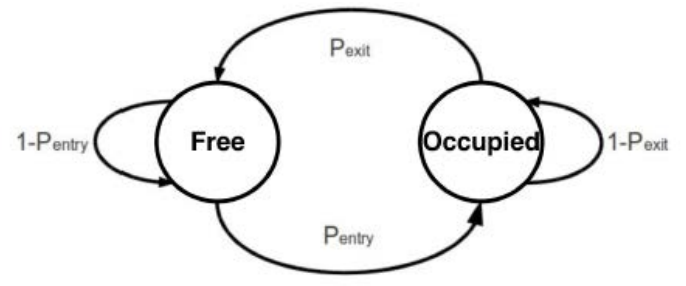

Fig. 1. State transition of a grid of IMAC. Each grid is a two state Markov chain being in Free or Occupied. The grid changes its state from Free to Occupied with probability of $\mathrm{P}_{\text {entry }}$; from Occupied to Free with probability of $\mathrm{P}_{\text {exit }}$

\subsection{Independent Markov Chain Occupancy Grid Map (IMAC)}

The IMAC ${ }^{[5]}$ is an occupancy grid map based on the proposed model of Luber ${ }^{[4]}$. Each grid has two states, which being in occupied or free. Assuming that the state transition of each grid is independent and thus it can be modeled as a Poisson process. Fig.1 shows the Poisson process state transition graph of each grid and the probabilities. These state transition probability are estimated as follows. The estimated state transition probability of grid $m$ is $\left(\hat{\lambda}_{m, \text { exit }}, \hat{\lambda}_{m, \text { entry }}\right)$, $\hat{\lambda}_{\text {m,exit }} \sim P_{m, \text { exit }}=p\left(m_{t}=\right.$ freel $m_{t-1}=$ occupied $)$

$\hat{\lambda}_{m, \text { entry }} \sim P_{m, \text { entry }}=p\left(m_{t}=\right.$ occupied $\mid m_{t-1}=$ free $)$.

After we got the observations for grid $m$, calculate

$\hat{\lambda}_{\text {m,exit }}=\frac{\text { \# events }: \text { occupied to f ree }+1}{\text { \# observations }: \text { when occupied }+1}$ $\hat{\lambda}_{m, \text { entry }}=\frac{\text { \# events }: \text { f reeto occupied }+1}{\text { \# observations }: \text { when } f \text { ree+ } 1}$

where \#events:occupied to free is the number of times a grid is observed turning from occupied to free, \#observations when occupied is the number of observations done in occupied state and \#events:free to occupied. \#observations when free are the respective quantities for observing a grid turning from free to occupied and observing the grid in free state. The interpretation of $\hat{\lambda}_{m \text {,exit }}$ as a Poisson rate parameter is the expected number of state change events per observation, given that we are in occupied state.

The experiment in a warehouse Saarinen et al. ${ }^{[5]}$ shows that we can archive a good grid map of dynamic environment by using the IMAC model.

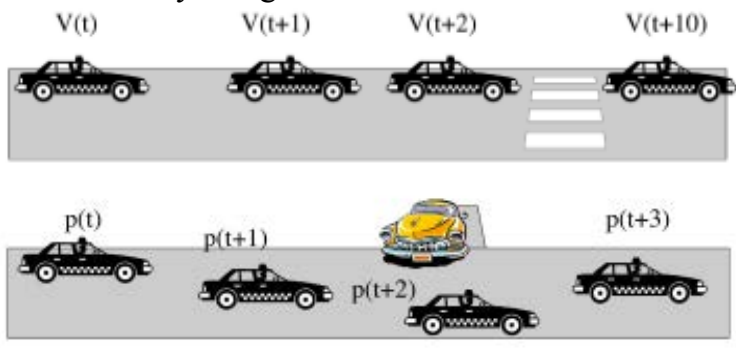

Fig. 2. Example of stop by incident.

\subsection{Grid Update Algorithm and Driving Behavior}

This sub-section describes algorithm to estimate grid states from GPS log data, and method to detect and create map of incident stops. Fig. 2 shows two examples of incident spots on traffic road. At the zebra crossing line, vehicle has to stop for a while $(v(t+2)=0)$. After that, it starts to increase its speed $(\mathrm{v}(\mathrm{t}+10)>\mathrm{v}(\mathrm{t}+2))$. The lower part of Fig. 2 shows an example of stop by obstacle.

We assume that if a vehicle decreases its speed, there must be an incident event around its location. Otherwise, if a vehicle drives smoothly, there are no incident events interfering with driving process. The algorithm 1 shows the proposed algorithm.

Algorithm 1. Grid property update algorithm

\begin{tabular}{ll}
\hline 1 & if vehicleIsStop $(t)==$ true then \\
2 & for all grid $q$ in map do \\
3 & for all grid $m: \mid$ m in neighbor $\left(x_{i}\right.$, span $\left._{\text {min }}\right)$ do \\
4 & Increase number of observations in occupancy \\
& by $w\left(x_{i}(t) ; q\right)$ \\
5 & if vehicleIsStopi $(t-1)==$ false then \\
6 & Increase number of entry event by $w\left(x_{i}(t) ; m\right)$
\end{tabular}




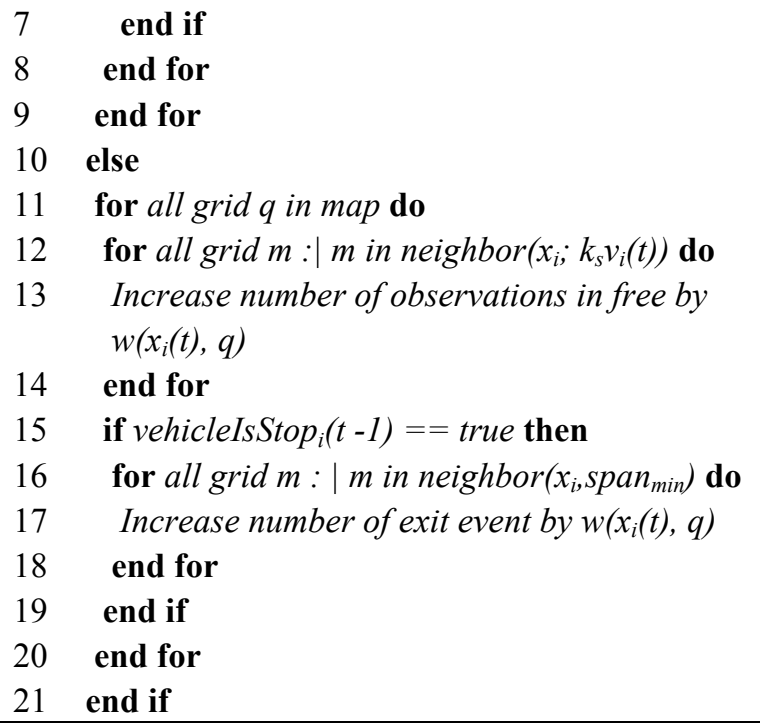

where neighbor $(x, d)$ means the set of grids of $d$ far from $\mathrm{x}, v_{i}(t)$ in line 12 is speed of vehicle $i$ at $t$, and $k$ is a constant. This algorithm updates the grid by using vehicle states as follows. We suppose that a vehicle drives slowly according to its distance to obstacle. GPS $\log$ data contains lot of noise, thus the proposed algorithm tries to update states of neighboring grids around the vehicle location. As shown in Fig.3, if a vehicle stops the occupied observations and transition events of grids around the vehicle location are updated by using weight $w$. Otherwise, the free observations and transition events of grids are updated.

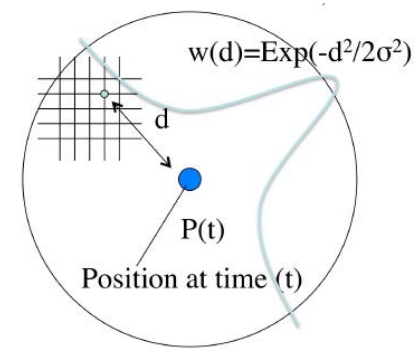

Fig. 3. GPS noise solution (circle update)

\subsection{Proposed Method for Anomaly Stop Detection}

In this sub-section, we proposed a simple algorithm to detect anomalous stop by using the acquired grid map described in 2.2. For detecting anomaly stop time, we evaluate the stop time.

Algorithm 2 shows the proposed algorithm. The probability of occurrence of a given stop time $\mathrm{P}_{\mathrm{a}, \mathrm{t}}$ can be calculate by the two-Markov chain in Fig.1.
Algorithm 2. Probability calculation algorithm

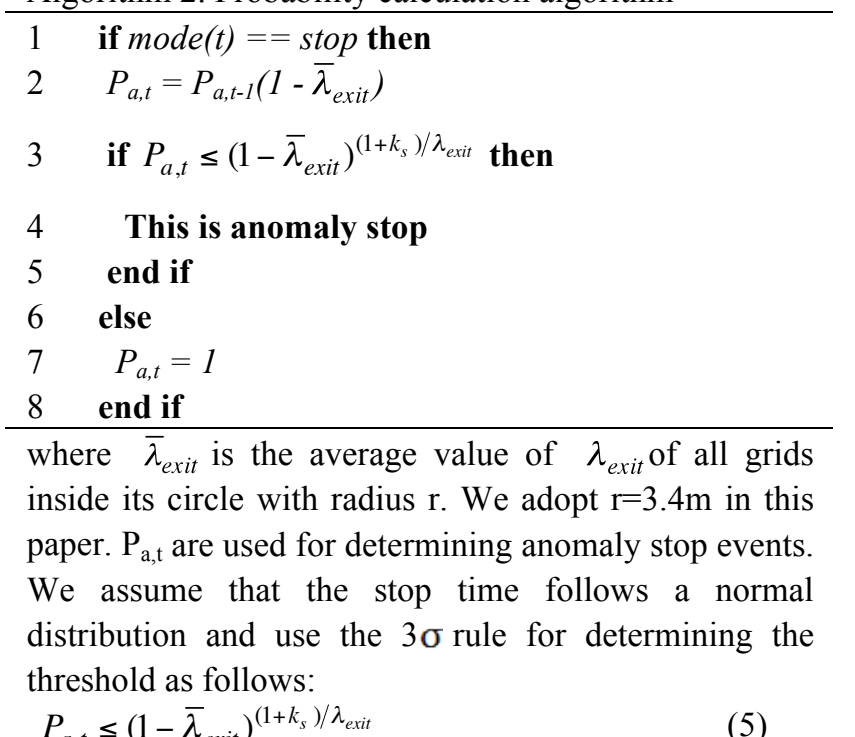

If a stop events that satisfy the Eq. (5) then it is said to be an anomaly stop event. $k_{s}$ is an constant parameter. $\left(1+k_{s}\right) / \lambda_{\text {exit }}$ is the stop time which has $k_{s} \sigma$ steps far from the average. In the experiment, we set $k_{s}=3$.

\section{Experiment}

In this section, we evaluate the proposed model by a real world experiments. The first experiment is for map generation; the second experiment is for anomaly stop detection. We use an Apple iPhone 4s for recording GPS log data. The log data includes GPS location, heading, and time series which are recorded at frequency of $100 \mathrm{~Hz}$. The vehicle is a Mazda6 20c, the smartphone was put on vehicle door pocket.

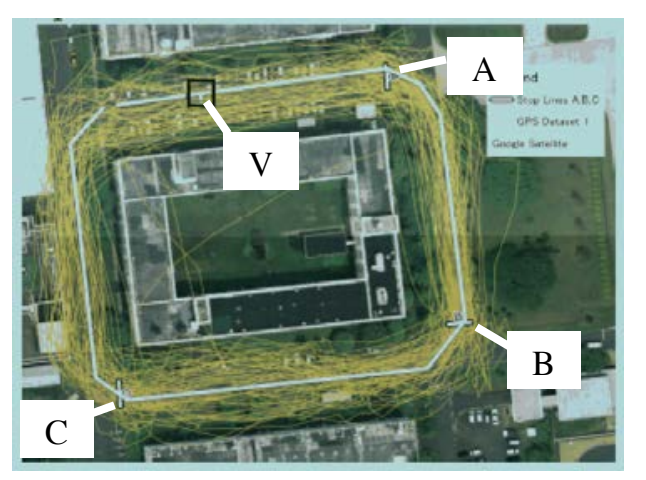

Fig.4 Dataset1 for map generation. The solid rectangle is a clockwise test track for the vehicle. The GPS data is shown by the set of thick yellow lines. In Dataset1, there are three stop lines A, B, C which the vehicle stops at 10 seconds, 6 seconds, and 4 seconds respectively. The red box is the virtual stop location $\mathrm{V}$ for anomaly detection test. 


\subsection{Map Generation}

The objective of this experiment is to evaluate if the proposed method can be used to create map by using real world data. Fig.4 shows the test track which represent as the white line. The vehicle drives around a rectangle building follow clockwise. The white lines with black bound are temporarily stop lines A, B, and C. Stop line $\mathrm{V}$ is an anomaly stop line used for the next experiment.

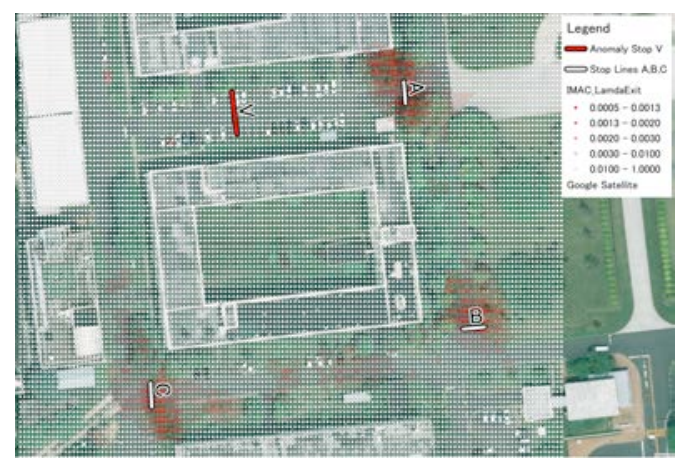

Fig. 5. Grid map of $\lambda_{\text {exit }}$ of half of Datasetl (red-white dots). There is no transition events (occupied to free or free to occupied) happened at white grids. In others hand, there are many transition events happened at red grids, especially at the stop line A, B, C.

We use two datasets for evaluating our method which has the same test track. In Dataset1, there are three stop lines A, B, C at which vehicle stops for 10 seconds, 6 seconds, and 4 seconds. Dataset2 has three stop lines as in Dataset1 plus stop line $\mathrm{V}$ at which the vehicle stops for 10 seconds. These two datasets include 70 rounds with $5 \mathrm{~km}$. Fig. 4 shows Dataset1. Despite of passing the same path on the all laps, the both datasets include a lot of noise which make the GPS paths is out of the test track when installed in vehicle door pocket.

The grid map of $\lambda_{\text {exit }}$ based on half of Dataset1 is shown in Fig. 5. The $\lambda_{\text {exit }}$ of each grid is presented as a RED-WHITE gradient color (RED $\sim$, WHITE $\sim$ ). At each center of the stop lines A, B, C, the values of $\lambda_{\text {exit }}$ are smaller than neighbor grid. Also, the colors around the stop line A are redder than those of the stop lines B, C. In other words, the vehicle stops at the stop line A longer than at the stop line B, C. It means that the grid map reflects Dataset 1 correctly.

This experiment result shows that the acquired grid map is suitable with those of the experiment setup.

\subsection{Anomaly Stop Detection}

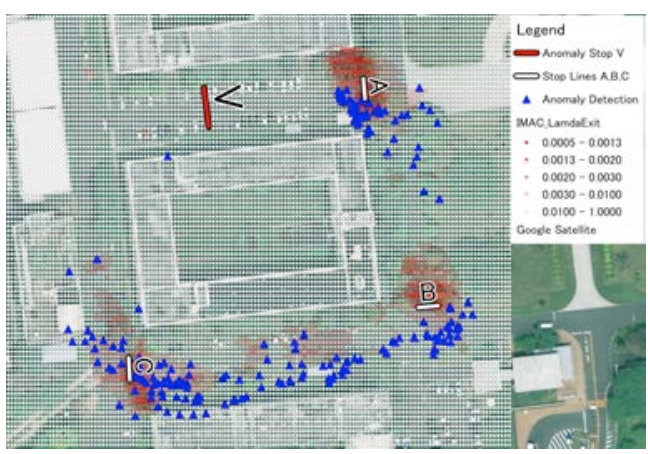

Fig. 6. Grid map of $\lambda_{\text {exit }}$ of half of Dataset1 and anomaly stop detection by the rest half of Dataset1. The blue triangles show the anomaly stop detection of the same dataset for map generation and anomaly stop detection. There is no anomaly stop at the location V. However, there are many wrong anomalous stop are detected at the stop line A, B, C because of GPS noise.

This section carries out anomaly stop detection experiment. We use half of Dataset1 to create grid map. Then half of Dataset2 (which the vehicle stops 10 seconds at stop line V additionally) is tested as dataset may have anomaly stops. We execute the following two combinations of anomaly detection trials for evaluating accuracy of the proposed method.

We set up the following scenarios:

S1: Map generation dataset is $50 \%$ of Dataset1. The test dataset for anomaly is the remaining $50 \%$ of Dataset1.

S2: Map generation dataset is $50 \%$ of Dataset1. The test dataset for anomaly stop detection is $50 \%$ of Dataset 2 .

The purpose of scenario S1 is to check the validity of the proposed model. Because the map generation dataset and the anomalous stop detection dataset uses the same source, it is expected not to show any anomaly stop. The purpose of scenario S2 is to detect anomalous stop.

The results of the anomaly stop detection are shown in Fig.6 and Fig.7. The result of S1 is shown in Fig.6. The result of S2 is shown in Fig.7. The triangles are the anomaly stop locations detected by the proposed method. No anomaly stop is detected around the location $\mathrm{V}$ in S1. There are many anomaly stops detected around the location $\mathrm{V}$ in $\mathbf{S 2}$.

Table 1 shows the anomaly detection ratio for two scenarios $\mathrm{S} 1$ and $\mathrm{S} 2$ at the location V. Ideally, the maximum of the number of anomaly stops is the number of data during stop at location $\mathrm{V}$, namely, the number of laps $\times 10 \mathrm{sec} \times 100 \mathrm{~Hz}$. TP(True Positive) column in this table indicates the ratio of successfully 
detected anomaly stops. $r_{v}$ is distance far from stop line $\mathrm{V}$. We adopt $\mathrm{r}_{\mathrm{s}}=4.7 \mathrm{~m}$ and $9.4 \mathrm{~m}$. $4.7 \mathrm{~m}$ is equal to the car length. The false detected anomaly stops are others anomaly stop more far from stop line $\mathrm{V}$.

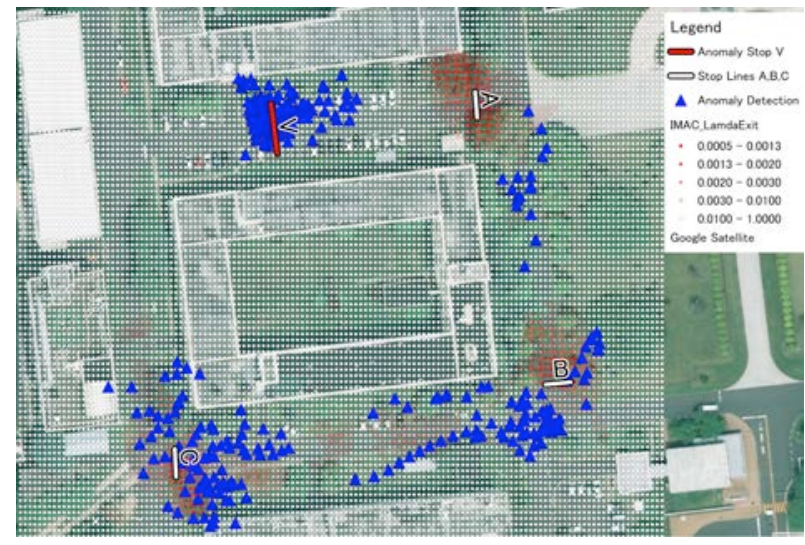

Fig. 7. Grid map of $\lambda_{\text {exit }}$ of half of Dataset1 and anomaly stop detection by half of Dataset2. The blue triangles show the anomaly stop detection. The proposed algorithm detects many anomaly stop at the virtual stop line $\mathrm{V}$

The TP of detection rate of $\mathrm{S} 1$ is $0 / 0$ because there is no anomaly stop at the location $\mathrm{V}$ in the dataset. Otherwise, the TP of detection rate of $\mathrm{S} 2$ is $\mathrm{TP}=0.399$ for $\mathrm{r}_{\mathrm{v}}=4.7 \mathrm{~m}$ and $\mathrm{TP}=0.642$ for $\mathrm{r}_{\mathrm{v}}=9.4 \mathrm{~m}$. It means that the large portion of the anomaly stops is located around stop line V. On the other hand, because of GPS noise, the vehicle speed is not always zero. As seen in Fig.7 there are other miss detections near the stop line $\mathrm{A}, \mathrm{B}, \mathrm{C}$. It suggests the more efficient method to filter the GPS noise is required which we consider as future work.

From the result, the proposed method can be used in the real world to detect anomaly stop with a reasonable accuracy.

Table 1. Anomalous stop detection rate (TP: True Positive, FP: False Positive, FN: False Negative, TN: True Negative)

\begin{tabular}{|c|c|c|c|c|}
\hline Scenario & TP & FP & FN & TN \\
\hline $\mathrm{S} 1$ & $0 / 0$ & 0 & 0 & 1 \\
\hline $\mathrm{S} 2\left(\mathrm{r}_{\mathrm{v}}=4.7 \mathrm{~m}\right)$ & 0.399 & 0.38 & 0.601 & 0.62 \\
\hline $\mathrm{S} 2\left(\mathrm{r}_{\mathrm{v}}=9.4 \mathrm{~m}\right)$ & 0.642 & 0.137 & 0.358 & 0.863 \\
\hline
\end{tabular}

\section{Conclusion}

This paper proposed a method for anomaly stop detection of a vehicle using smartphone log data. Despite of many researches of strong braking events are proposed, no works discussed on the discrimination of daily hard braking and anomaly breaking in relation with its geographic information. We proposed two algorithms. The first algorithm uses for estimating grid states by using GPS log data. The second algorithm uses for anomaly stop detection by using estimated grid map. In the anomaly detection method, we use the estimated grid map as ground truth map for comparing with test dataset. The anomaly stop detection method based on longer stop time is proposed. We also evaluated the proposed methods in a real world experiment. The results show that the estimated grid map and anomalous stop detection can be worked well in real world environment.

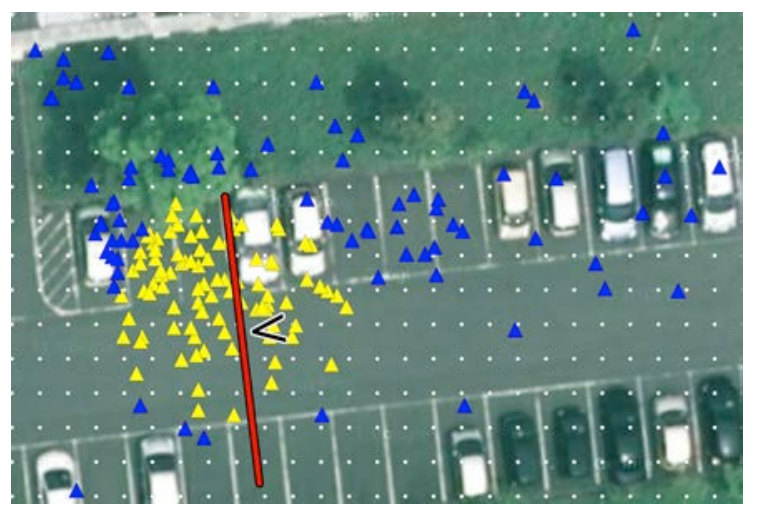

Fig. 8. Anomalous stop detection accuracy of scenario S2 (zoom details around the virtual stop line V). The blue and yellow triangles show all of anomalous stop detection around the virtual stop line $\mathrm{V}$. The yellow triangles are anomaly stops within $4.7 \mathrm{~m}$ radius circle from virtual stop line $\mathrm{V}$

\section{References}

1. J. C. Herrera, A. M. Bayen, Incorporation of Lagrangian measurements in freeway traffic state estimation, Transportation Research Part B 44 (2010) 460-481

2. B. N. Hilton, T. A. Horan, R. Burkhard and B. Schooley, SafeRoadMaps: Communication of Location and Density of Traffic Fatalities through Spatial Visualization and Heat Map Analysis Information Visualization (http://saferoadmaps.org/,2013)

3. The Honda safety project(http://safetymap.jp/,2013)

4. M. Luber, G. Diego Tipaldi, and K.O. Arras. Placedependent people tracking. The International Journal of Robotics Research, 30(3)(2011) 280-293.

5. J. Saarinen, H. Andreasson, and A. J. Lilienthal, Independent Markov chain occupancy grid maps for representation of dynamic environment, in Proc. 2012 IEEE/RSJ International Conference on Intelligent Robots and Systems (IROS, 2012), pp.3489 - 3495. 